\title{
A Multi-Scale Dynamic Mechanistic Model for Transient Analysis of PEFCs
}

\author{
Alejandro A. Franco ${ }^{a}$, Christian Jallut ${ }^{\mathrm{b}}$ and Bernhard Maschke ${ }^{\mathrm{b}}$ \\ ${ }^{a}$ Commissariat de l'Energie Atomique, DRT/LITEN/DTH/ \\ Laboratoire d'Essais et Validations de Composants $\mathrm{H}_{2}$-Piles à Combustible \\ 17, Rue des Martyrs FR-38000 Grenoble / France \\ alejandro.franco@cea.fr \\ ${ }^{b}$ Laboratoire d'Automatique et de Génie des Procédés \\ LAGEP UMR CNRS FR-5007 Université Lyon-1 \\ 43 Bd du 11 Novembre 1918 FR-69622 Villeurbanne cedex / France \\ jallut@lagep.univ-lyon1.fr \\ maschke@lagep.cpe.fr
}

\begin{abstract}
In this paper we propose a new knowledge model to understand the dynamic behavior of a Membrane-Electrodes Assembly (MEA) of a Polymer Electrolyte Fuel Cell. The model, multiscale and mechanistic, is based on irreversible thermodynamics and electrodynamics, and depends on the internal physical parameters such as the specific catalytic area, the electric permittivity of the materials, the reaction kinetics and the diffusion coefficients of the reactant species.

The model results of the coupling of a microscale transport phenomena description of charges (electrons and protons) through the electrode and the electrolyte thickness, a spatially distributed microscale model of the reactant diffusion (hydrogen and oxygen) through the Nafion ${ }^{\circledR}$ layer covering the Pt/C particles, and a spatially distributed nanoscale description of the Nafion ${ }^{\circledR}$-Pt interface. This interfacial model is based on an internal description of the electrochemical double layer dynamics, coupling the transport phenomena in the diffuse layer and the electrochemical reactions and water adsorption in the compact layer.

This multiscale model introduces many couplings between different physical domains, such as diffusionmigration transport and electrochemistry. A distributed-parameter Bond Graph description of the model has allowed to formulate it in a modular way, so that it could be adapted to other electrochemical systems (solid oxide fuel cells, Lithium-ion batteries...) or to be coupled witrh other physico-chemical phenomena (ageing mechanisms...).

The numerical resolution allows to simulate the MEA dynamics, depending on current, temperature, reactant pressures and structural composition of the electrodes (Pt and Nafion ${ }^{\mathbb{B}}$ loadings). Particularly, the model allows evaluating the sensitivity of impedance spectra to operating conditions and electrodes compositions, and it gives access to the contributions of the different phenomena and constitutive layers. $A$ qualitative experimental validation of the model was carried out on a specific bench and on single cells with different platinum loadings.
\end{abstract}

KEYWORDS: multi-scale model, irreversible thermodynamics, impedance spectroscopy diagnostics, electrochemical double layer, platinum/Nafion ${ }^{\circledR}$ loading optimization.

\section{NOMENCLATURE}

$\vec{D} \quad$ displacement vector $\left(\mathrm{C} \cdot \mathrm{m}^{-2}\right)$

$\vec{E} \quad$ electric field $\left(\right.$ N.C C $\left.^{-1}\right)$

$d$ thickness of a water molecule $\left(2 \times 10^{-10} \mathrm{~m}\right)$

$\vec{n} \quad$ number of dipoles opposed to the metallic phase per unit of area $\left(\mathrm{m}^{-2}\right)$

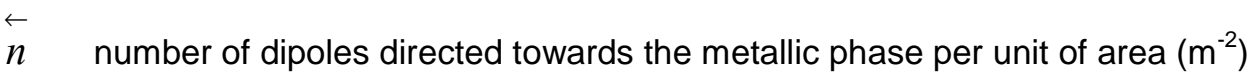

$n_{s} \quad$ number of free sites per unit of area of metallic phase $\left(\mathrm{m}^{-2}\right)$

$n_{H s} \quad$ number of sites covered by the intermediate reaction specie $H s$ per unit of area of metallic phase $\left(\mathrm{m}^{-2}\right)$

$n_{\mathrm{O}_{2} \mathrm{Hs}}$ number of sites covered by the intermediate reaction specie $\mathrm{O}_{2} \mathrm{Hs}$ per unit of area of metallic phase $\left(\mathrm{m}^{-2}\right)$

$n_{O H s} \quad$ number of sites covered by the intermediate reaction specie $O H s$ per unit of area of metallic phase $\left(\mathrm{m}^{-2}\right)$ 
$n^{*} \quad$ sum of free sites and sites covered by intermediate reaction species per unit of area of metallic phase $\left(\mathrm{m}^{-2}\right)$

$n^{\max } \quad$ maximal quantity of free sites per unit of area of metallic phase $\left(\mathrm{m}^{-2}\right)$

$T \quad$ absolute temperature $(\mathrm{K})$

$R \quad$ ideal gas constant $\left.\left(8.34 \mathrm{~J} \cdot \mathrm{K}^{-1} \cdot \mathrm{mol}^{-1}\right)\right)$

$\Delta G^{0} \quad$ Gibbs' free energy for the dipolar adsorption (J.mol $\left.{ }^{-1}\right)$

$\Delta G_{C}{ }^{0}$ dipolar chemical adsorption energy $\left(\mathrm{J} \cdot \mathrm{mol}^{-1}\right)$

$\Delta G_{e}{ }^{0}$ dipolar electrostatic adsorption energy (J. $\mathrm{mol}^{-1}$ )

$\Delta G_{i}{ }^{0}$ dipolar interaction adsorption energy $\left(\mathrm{J}^{\mathrm{mol}}{ }^{-1}\right)$

$a=2 \exp \left(-\Delta G_{C}^{0} / R T\right)$

$k=1.38 \times 10^{-23} \quad$ Boltzman's constant $\left(\mathrm{J}^{-1} \mathrm{~K}^{-1}\right)$

$F=96485 \quad$ Faraday's constant $\left(\mathrm{C} . \mathrm{mol}^{-1}\right)$

$f=F / R T$

$v_{i}$ electrochemical reaction rate $\left(\mathrm{mol} . \mathrm{s}^{-1} \cdot \mathrm{m}^{-2}\right)$

$k_{i}$ electronic standard rate constant $\left({\left.\mathrm{m} . \mathrm{s}^{-1}\right)}^{-1}\right.$

$J_{\text {Far }}$ faradaic current (A.m ${ }^{-2}$ )

$N_{A}=6.02214 \times 10^{23}$ Avogadro's number $\left(\mathrm{mol}^{-1}\right.$ )

$C_{i} \quad$ hydrogen or oxygen concentration (mol. $\mathrm{m}^{-3}$ )

$C_{H^{+}}$proton concentration (mol.m ${ }^{-3}$ )

$C_{F I X^{-}}$charged fixed sites concentration (sulfonates of Nafion $\left.{ }^{\circledR}\right)\left(\mathrm{mol}^{-3} \mathrm{~m}^{-3}\right)$

$D_{i} \quad$ diffusion coefficient of hydrogen or oxygen in Nafion ${ }^{\circledR}\left(\mathrm{m}^{2} \cdot \mathrm{s}^{-1}\right)$

$D_{H^{+}}$diffusion coefficient of protons in Nafion ${ }^{\circledR}\left(\mathrm{m}^{2} \cdot \mathrm{s}^{-1}\right)$

$J_{H_{2}} \quad$ molar flux of hydrogen in Nafion ${ }^{\circledR}\left(\mathrm{mol}^{-1} \cdot \mathrm{m}^{-2}\right)$

$J_{H^{+}}$molar flux for the protons in Nafion ${ }^{\circledR}\left(\mathrm{mol}^{-1} \cdot \mathrm{s}^{-2}\right)$

$I$ total current demanded to the fuel cell (A)

$J$ local electronic current density at the surface of the Pt/C phase (nanoscopic model) (A.m ${ }^{-2}$ )

$S_{\text {electrode }}$ geometric surface of the Membrane-Electrodes Assembly (MEA) $\left(\mathrm{m}^{2}\right)$

$p_{i} \quad$ hydrogen or oxygen partial pressure in the pore phase (pascal)

$g_{H^{+}}$protonic conductivity $\left(\Omega^{-1} \cdot m^{-1}\right)$

$g_{e^{-}}$electronic conductivity in the $\mathrm{Pt} / \mathrm{C}$ phase $\left(\Omega^{-1} \cdot m^{-1}\right)$

\section{Greek letters}

$\eta$ potential difference at the metal/ electrolyte interface (through the compact layer) (V)

$\psi$. electric potential in the metallic phase $(\mathrm{Pt} / \mathrm{C})(\mathrm{V})$

$\phi$. electric potential in the electrolyte phase $(\mathrm{V})$

$\sigma$ charge surface density $\left(\mathrm{C} . \mathrm{m}^{-2}\right)$

$\varepsilon_{0}$ electric permittivity of vacuum $\left(8.85 \times 10^{-12} \mathrm{C}^{2} \cdot \mathrm{N}^{-1} \cdot \mathrm{m}^{-2}\right)$

$\varepsilon_{C \in}$ electric permittivity in the compact layer $\left(\mathrm{C}^{2} \cdot \mathrm{J}^{-1} \cdot \mathrm{m}^{-1}\right)$

$\varepsilon_{C D}$ electric permittivity in the diffuse layer (electrolyte phase) $\left(C^{2} \cdot \mathrm{J}^{-1} \cdot \mathrm{m}^{-1}\right)$

$v_{\theta}$ magnetic permeability of vacuum $\left(4 \pi \times 10^{-7} \mathrm{~kg} . \mathrm{m} \mathrm{C}^{-2}\right)$

$\Gamma$ interfacial dipolar surface density (debye. $\mathrm{m}^{-2}$ )

$\mu$ dipolar moment of a water molecule $\left(0.62 \times 10^{-29} \mathrm{C} . \mathrm{m}\right)$ 


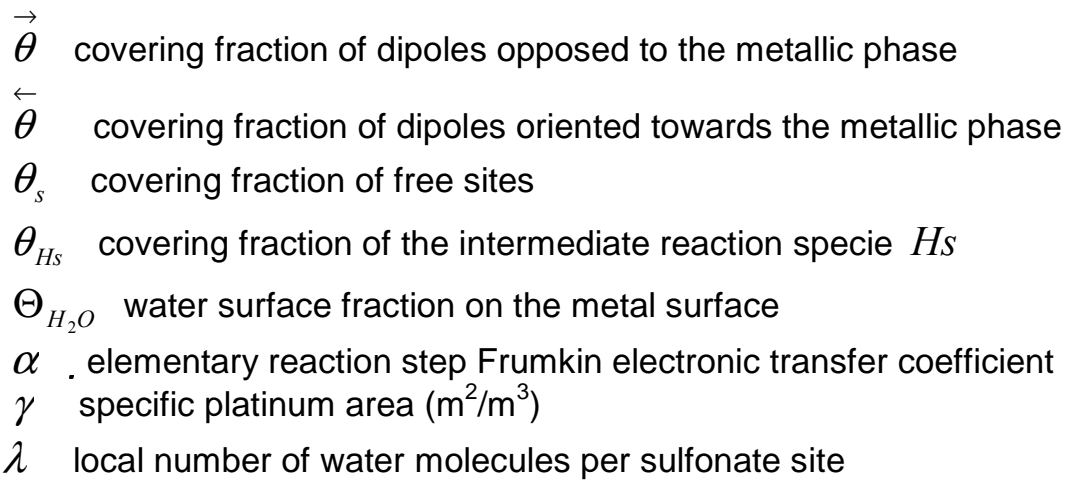

\section{Introduction}

The electrochemical impedance spectroscopy (EIS) is a widely used experimental technique for the transient analysis of Polymer Electrolyte Fuel Cells (PEFC) [1]. Experimental results are usually analyzed using analogical AC linear circuit models [2-3]. In these models, charge transfer is typically represented by ideal resistors and mass transport by complex impedances. The interfacial accumulation of intermediate reaction species and the electrochemical double layer at the metal/electrolyte interface (for instance, Nafion ${ }^{\circledR} / \mathrm{Pt}$ ) are represented by uncoupled capacitive elements [3-5].

The parameters of these models have to be fitted at each electrodes working point (given by the nominal current, the temperature and the reactant species concentration). For instance in the case of PEFC electrodes, it is experimentally known that the character of EIS spectra strongly depends on these working conditions. Also, due to the fact that the AC circuit components are a global linear representation of the nanoscopic physical mechanisms involved, an analogical circuit involving three or more circuit elements can often be arranged in various ways and still produce similar impedance responses. Other authors [3] derive analytical expressions of the impedance dependence with nominal current, supposing simple electrochemical mechanisms in the electrodes: the analytical expressions obtained are not reusable (they have to be recalculated if one changes a mechanism or couple it with other physicochemical phenomena); furthermore, analytical computations of complex impedances become impossible for more complicated mechanisms.

In this paper we propose a multi-scale mechanistic Membrane-Electrodes Assembly (MEA) dynamic model [6-8] based on the distributed-parameter Bond Graph language [8-14] (irreversible thermodynamics and electrodynamics) and depending on the internal physical parameters.

Our model is composed of an anodic and cathodic electrodes and an membrane electrolyte mechanistic submodels (the electrodes of some micrometers thickness are separated by a 20 to 100 micrometer thick membrane). The electrodes are constituted of catalyst/electronic conductor particles (Pt/C phase) (of 100 nanometers size, and supposed to be uniformly distributed in the electrode volume) immersed in a protonic conductor medium (Nafion ${ }^{\oplus}$ phase) (of maximum $10^{-7} \mathrm{~m}$ thickness) and a void fraction (pore phase) (Fig.1). An electrochemical double layer is formed at the nanometric vicinity of the $\mathrm{Pt} / \mathrm{C}$ phase (Fig. 2). The multiscale model results of the coupling of a microscale proton and electron transport description through the electrode thickness, a spatially distributed microscale model of the reactant $\left(\mathrm{H}_{2}\right.$ in the anode, $\mathrm{O}_{2}$ in the cathode) diffusion through the Nafion ${ }^{\circledR}$ layer, and a spatially distributed nanoscopic model of the $\mathrm{Pt} / \mathrm{C}$ Nafion ${ }^{\circledR}$ interface, introduced by Franco et al. elsewhere [15]. The last one is constituted of a diffuse layer and a compact layer. The diffuse layer is composed of spatially moving proton ions, of counter-ions representing the negatively charged and spatially fixed sulfonate groups of the Nafion ${ }^{\circledR}$ phase, and of water molecules. The compact layer is formed by adsorbed water molecules at the surface of the Pt/C phase, and intermediate reaction species of the electrochemical reactions (converting $\mathrm{H}_{2}$ into protons and electrons in the anode, and $\mathrm{O}_{2}$, protons and electrons into water in the cathode). In a first step, we consider here the case of isothermal fuel cells fed with pure hydrogen and oxygen fully saturated with water vapour. Additional water production is evacuated through the liquid phase. In these conditions it appears not necessary to take into account the gas diffusion layers of a typical fuel cell [7-8]. The model computes dynamically the potential difference between the two electrodes in response to a time dependent current demand $I(t)$ (and depending on $P_{A}, P_{C}$-total anodic and cathodic pressures in the pore phase- and $T$-PEFC temperature-). 


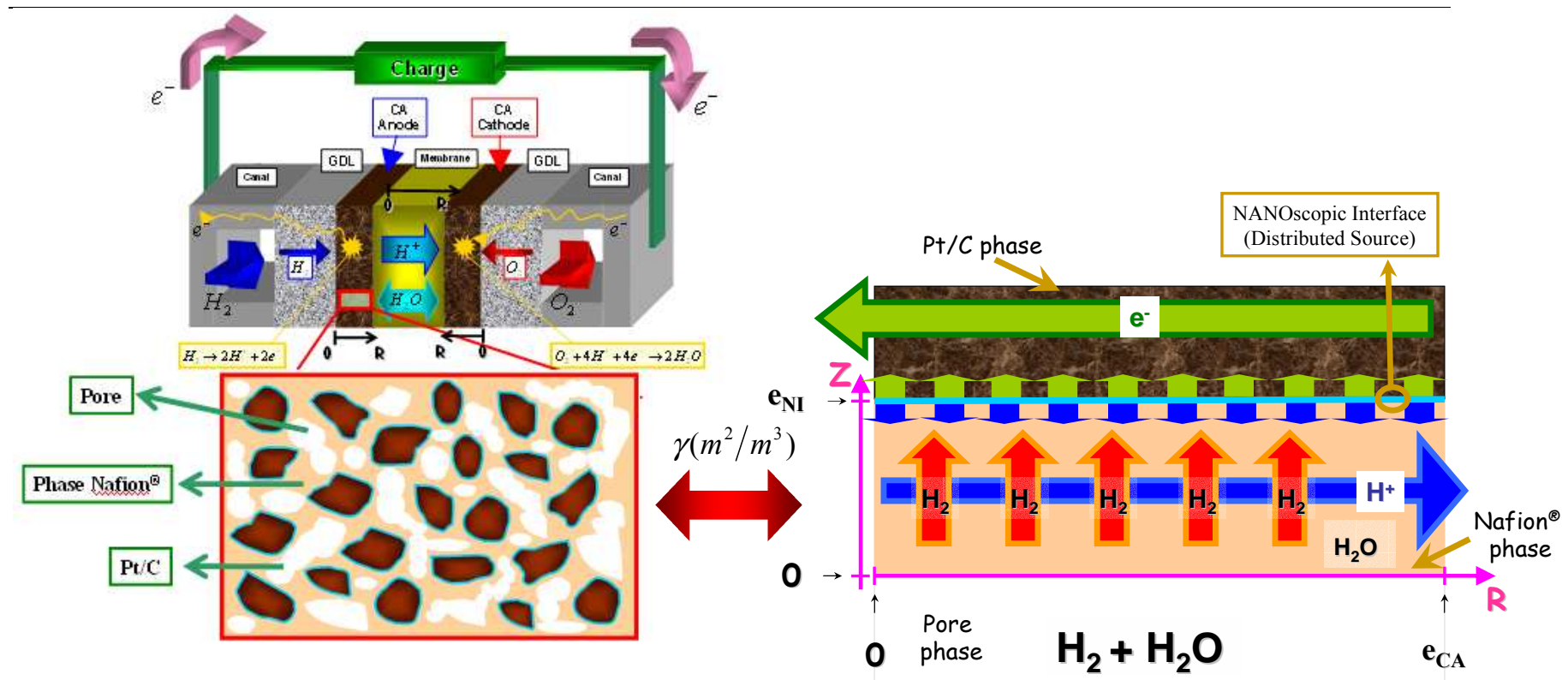

Fig. 1: The electrode morphology and the multi-scale model of the volumetric electrode (example of the anode) and localization of the nanoscopic interface model. The reactant specie (hydrogen) diffuses through the impregnated Nafion ${ }^{\circledR}$ layer, protons and electrons are produced at the nanoscopic interface, and evacuated through the electrode thickness.
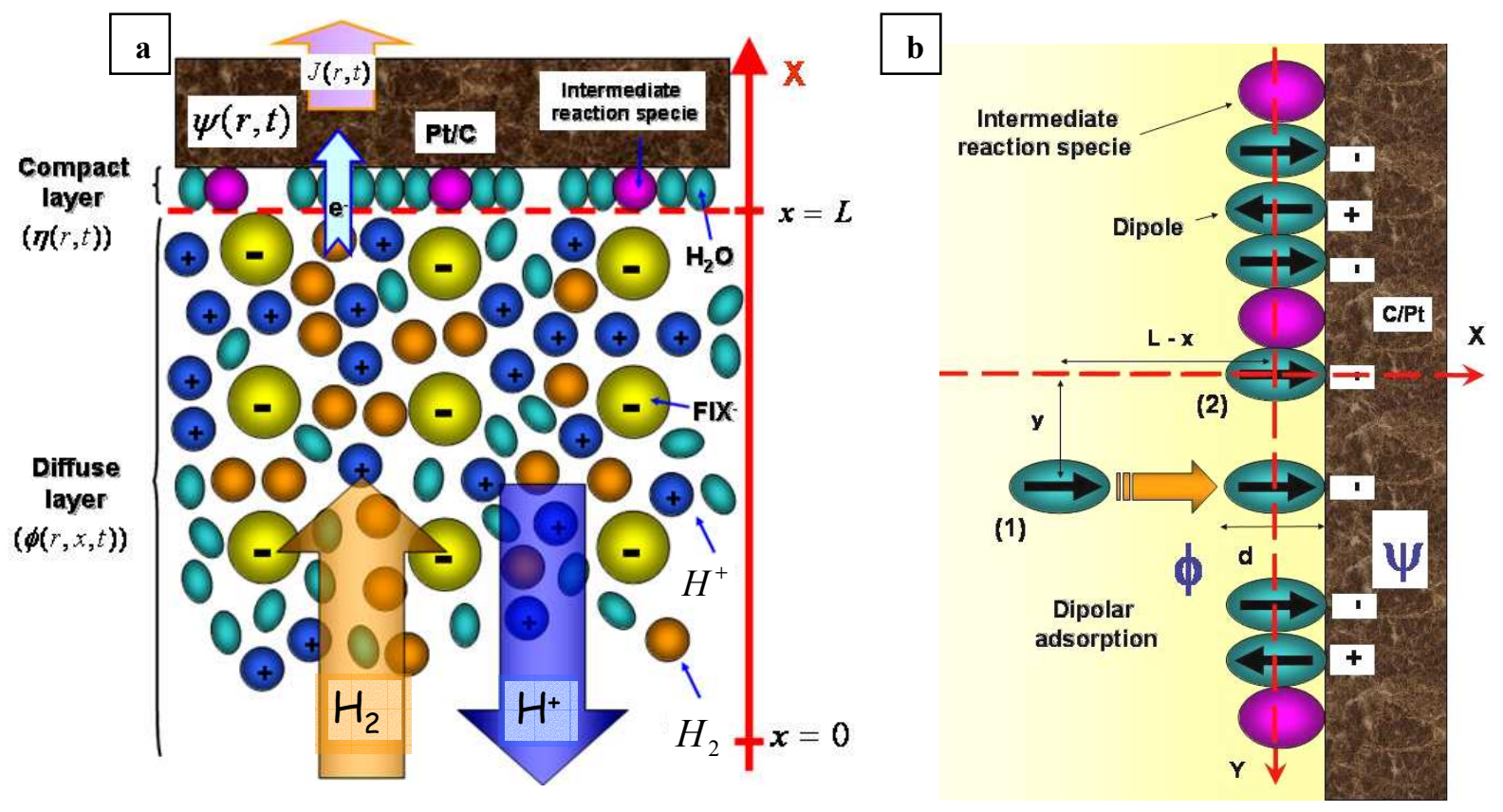

Fig. 2: a) A schematic representation of the nanoscopic interface model (anodic case): the reactant specie (hydrogen) arrives to the compact layer where the electron transfer reaction takes place. Protons are produced at $x=L$ and evacuated through $x=0$. b) Intermediate reaction species and dipolar water adsorption on the Pt surface (cf. Section 3).

\section{The micro-scale sub-models}

The reactant (noted by $i$ ) transport through the impregnated Nafion ${ }^{\circledR}$ layer thickness is given by:

$$
\vec{J}_{i}=-\left(C_{i} D_{i} / R T\right) \nabla_{z} \mu_{i} \text { and } \partial C_{i} / \partial t=-\nabla_{z} \cdot \vec{J}_{i}
$$

where $\mu_{i}(r, z, t)$ is the chemical potential of the $i$ specie, $C_{i}(r, z, t)$ is the molar concentration (mol. $\mathrm{m}^{-3}$ ), $J_{i}(r, z, t)$ the molar flux $\left(\mathrm{mol} \cdot \mathrm{m}^{-2} \cdot \mathrm{s}^{-1}\right), D_{i}$ the diffusion coefficient in the Nafion ${ }^{\circledR}$ phase, $T$ the temperature (K) and $R$ the ideal gas constant. 
The local electronic current $i_{e^{-}}(r, t)$ in the $\mathrm{Pt} / \mathrm{C}$ phase at the microscale is given by:

$$
i_{e^{-}}(r, t)=-S_{M E A} g_{e^{-}} \nabla_{r} \psi(r, t) \text { and } \nabla_{r}\left(i_{e^{-}}(r, t) / S_{M E A}\right)=S_{e^{-}}(r, t)
$$

where $\psi(r, t)$ is the local electronic electrical potential, computed by the nanoscopic sub-models, $g_{e^{-}}$is the electronic conductivity and $S_{M E A}$ is the MEA surface according the direction $R$. The local production or consummation is given by $J(r, t) \gamma=S_{e^{-}}(r, t)$, where $J(r, t)$ is the current density $\left(\mathrm{A} / \mathrm{m}^{2}\right)$ - boundary condition at the nanoscale, and where $\gamma$ is the specific platinum area (in $\mathrm{m}^{2} \cdot \mathrm{m}^{-3}$ ) (cf. Fig.1). The boundary conditions for (2) are $i_{e^{-}}\left(r=e_{C A}, t\right)=0$ and $i_{e^{-}}(r=0, t)=I(t)$ (demanded global current, input of the model). As showed by Franco et al. elsewhere, at the microscopic scale the electroneutrality for protons in the Nafion ${ }^{\circledR}$ phase can be assumed because we are far of the electrified surface (Pt/C-Nafion ${ }^{\circledR}$ nanoscopic interface) [68] [15], so the protonic current is given by:

$$
i_{H^{+}}(r, t)=-S_{M E A} g_{H^{+}} \nabla_{r} \phi(r, t) \text { and } \nabla_{r}\left(i_{H^{+}}(r, t) / S_{M E A}\right)=S_{H^{+}}(r, t)
$$

where $\phi(r, t)$ the local protonic electrical potential in the Nafion ${ }^{\circledR}$ phase and $g_{H^{+}}$the protonic conductivity. The proton production (anode) and consummation (cathode) are given by the distributed source $S_{H^{+}}(r, t)=J(r, t) \gamma$, where $J(r, t)$ is calculated by the microscopic electron transport model.

\section{The nanoscale sub-models}

The diffuse layer part of the nanoscale sub-model (cf. Fig.2a) takes into account the transport by diffusion and electrical migration of reactants, depending on the electric field generated by the resulting charge distribution. All the species $\left(H^{+}, H_{2}, O_{2}, F I X^{-}\right.$and water) are supposed to be punctual (interparticle interaction neglected). Solvatation by water and convection are not considered here. The reactant transport is treated as in the microscopic model (cf. equation (1)). The $H^{+}$transport close to the interface Pt/C-Nafion ${ }^{\circledR}$ is governed by (electroneutrality cannot be assumed [6-8] [15]):

$$
\begin{gathered}
-\left(D_{H^{+}} C_{H^{+}} / R T\right) \nabla_{x} \widetilde{\mu}_{H^{+}}=J_{H^{+}} \text {and } \partial C_{H^{+}} / \partial t=-\nabla_{x} . J_{H^{+}} \\
\left(F / \varepsilon_{C D}\right)\left(C_{H+}-C_{F I X}\right)=-\nabla_{x}{ }^{2} \phi+\varepsilon_{C D} v_{0} \partial^{2} \phi / \partial^{2} t
\end{gathered}
$$

where $\widetilde{\mu}_{H^{+}}=\mu_{H^{+}}+F \phi$ (electrochemical potential), $\phi(r, x, t)$. is the electrical potential in the electrolyte (neglecting the magnetic effects [8] [15]) and $C_{F I X}$ the sulfonate concentration in the Nafion ${ }^{\circledR}$ electrolyte.

At the compact layer level, $x=L$ (cf. Fig. 2a), the Frumkin's overpotential [6-8] [15] allows the development of the electrochemical reaction on the Pt surface. This potential discontinuity is given by:

$$
\eta(r, t)=\psi(r, t)-\phi(r, x=L, t)
$$

According to the superposition principle [7-8] [15], $\eta(r, t)$ is also given by:

$$
\psi(r, t)-\phi(r, x=L, t)=\eta(r, t)=-\left(\sigma / \varepsilon_{C C}\right) d+\Gamma(\sigma) / \varepsilon_{C D}
$$

where the first term is linked to the thickness of the compact layer $d$, and the second one to its dipolar nature. In (7), $\sigma$ is the charge surface density on $\mathrm{Pt}, \varepsilon_{C C}$ is the electric permittivity of the compact layer, $\varepsilon_{C D}$ the electric permittivity of the diffuse layer (supposed independent of temperature) and $\Gamma$ is the surface dipolar density (in debye $/ \mathrm{m}^{2}$ ) given by $\Gamma=\mu(\vec{n}-\stackrel{\leftarrow}{n})$ where $\mu$ is the dipolar moment of a water molecule, $\vec{n}$ (respectively $\stackrel{\leftarrow}{n}$ ) the number of dipoles per surface unit towards (respectively opposed) to the metal (cf. Fig.2b). The oriented adsorption of molecules is described here by the two equilibrium reactions $\mathrm{H}_{2} \mathrm{O}+s \leftrightarrow \stackrel{\leftarrow}{\mathrm{H}_{2}} \mathrm{O}-s$, where $s$ is a free site of adsorption on the metal surface. The application of the mass action law gives: 


$$
\underset{n}{\stackrel{\rightleftarrows}{n}} /\left(n_{s} \Theta_{H_{2} O}\right)=\stackrel{\vec{K}}{K}=\exp [-\stackrel{\vec{\leftarrow}}{0} / R T]=\exp \left[-\left(-\Delta G_{C}^{0} \mp \frac{\mu \sigma}{\varepsilon_{C C}} \mp \frac{A \mu^{2}}{d^{3}}\left(\frac{\vec{n}-\bar{n}}{n^{*}+\vec{n}+\bar{n}}\right)\right) / R T\right]
$$

where $n_{s}$ is the number of free sites per surface unit, $\Theta_{H_{2} O}$ the activity of the surface free water (supposed to be constant), and $\underset{\Delta G^{0}}{\vec{\leftarrow}}$ is the sum of the Gibb's free energies associated to the adsorptions: the first term represents the chemical energy of adsorption (supposed to be independent of the dipolar orientation), the second one the electrostatic energy corresponding to the necessary work to bring a dipole from infinity to the surface, and the third one the interdipolar interaction energy. $n^{*}$ denotes the sum of free sites and occupied sites by intermediates per surface unit. We can demonstrate [15] that from (8) we can write $\Gamma=-a n_{s} \Theta_{H_{2} O} \mu \operatorname{Sinh}[X], \quad$ with $\quad X=\left(\mu /\left(k T \varepsilon_{C L}\right)\right)+\left(A \mu^{2}\right) /\left(k T d^{3}\right)\left(\vec{n}-\stackrel{\leftarrow}{n} / n^{*}+\vec{n}+\stackrel{\leftarrow}{n}\right) \quad$ and $\quad a=2 \exp \left(-\Delta G_{C}^{0} / R T\right)$ ( $A=\zeta[3] / 2 \pi \varepsilon_{C D}, \zeta$ being the Riemann's function). $\mathrm{X}(\sigma)$ is calculated from:

$$
\operatorname{aSinh}[X] /\left(\frac{n^{*}}{n_{s} \Theta_{\mathrm{H}_{2} \mathrm{O}}}+a \operatorname{Cosh}[\mathrm{X}]\right)=\sigma\left(d^{3} / \varepsilon_{C C} A \mu\right)-\mathrm{X}\left(k T d^{3} / A \mu^{2}\right)
$$

Finally, we obtain for the Frumkin's overpotential:

$$
\eta(r, t)=-\frac{\sigma}{\varepsilon_{C C}} d-\frac{a n_{s} \Theta_{\mathrm{H}_{2} \mathrm{O}} \mu \operatorname{Sinh}[\mathrm{X}]}{\varepsilon_{C D}}
$$

A Tafel-Heyrovsky-Volmer reaction mechanism [6-8] [11-15] for the anodic hydrogen oxidation is used to calculate the temporal evolution of $n^{*}$ (and so of $n_{s}$ ) in (9) and (10):

$$
\begin{array}{rrr}
H_{2}+2 s \leftrightarrow \rightarrow 2 H s & (\text { Tafel }) & v_{T A F}=k_{T A F} \theta_{s}^{2} C_{H_{2}}(r, L, t)-k_{-T A F} \theta_{H s}^{2} \\
s+H_{2} \leftrightarrow \rightarrow H s+H^{+}+e^{-} & \text {(Heyrovsky) } & v_{H E Y}=k_{H E Y} \theta_{s} C_{H_{2}}(r, L, t) e^{\left(1-\alpha_{H E Y}\right) f \eta}-k_{-H E Y} \theta_{H s} C_{H^{+}}(r, L, t) e^{-\alpha_{H E Y} f \eta} \\
H s \leftrightarrow \rightarrow s+H^{+}+e^{-} & (\text {Volmer }) & v_{V O L}=k_{V O L} \theta_{H s} e^{\left(1-\alpha_{V O L}\right) f \eta}-k_{-V O L} \theta_{s} C_{H^{+}}(r, L, t) e^{-\alpha_{V O L} f \eta}
\end{array}
$$

where $v_{T A F}, v_{H E Y}$ and $v_{V O L}$ are the associated reaction step rates written as functions of $\eta(r, t)$. In these expressions $\theta_{s}$ the surface coverage by free sites, $\theta_{H s}$ the surface coverage by the monoatomic hydrogen adsorbed. $k_{i}$ and $k_{-i}$ are the standard kinetics constants, $\alpha_{i}$ the symmetry factors, $f=F / R T$ ( $F$ the Faraday's constant). In the cathode, a Damjanovic reaction mechanism [6-8] [11] [13] for the cathodic oxygen reduction is used:

$$
\begin{aligned}
& \mathrm{O}_{2}+H^{+}+e^{-}+s \leftrightarrow O_{2} H s(\operatorname{Dmj} 1) \quad v_{1}=k_{1} \theta_{s} C_{H^{+}}(r, L, t) C_{O_{2}}(r, L, t) e^{-\alpha_{1} f \eta(r, t)}-k_{-1} \theta_{O_{2} H s} e^{\left(1-\alpha_{1}\right) f \eta(r, t)}
\end{aligned}
$$

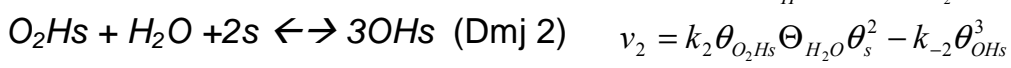

$$
\begin{aligned}
& \mathrm{OHs}+\mathrm{H}^{+}+e^{-} \leftarrow \rightarrow \mathrm{H}_{2} \mathrm{O}+\mathrm{s}(\mathrm{Dmj} 3) \quad v_{3}=k_{3} \theta_{\mathrm{OHs}} C_{H^{+}}(r, L, t) e^{-\alpha_{3} f \eta(r, t)}-k_{-3} \theta_{s} \Theta_{H_{2} O} e^{\left(1-\alpha_{3}\right) f \eta(r, t)}
\end{aligned}
$$

The surface coverages $\theta_{H s}, \theta_{\mathrm{O}_{2} \mathrm{Hs}}$ and $\theta_{\mathrm{OHs}}$ are given by the balance equations:

$$
\left.\begin{array}{l}
\left(n^{\max } / N_{A}\right)\left(d \theta_{H s} / d t\right)=-v_{V O L}+v_{H E Y}+2 v_{T A F} \\
\left(n^{\max } / N_{A}\right)\left(d \theta_{O_{2} H s} / d t\right)=v_{1}-v_{2} \\
\left(n^{\max } / N_{A}\right)\left(d \theta_{O H s} / d t\right)=3 v_{2}-v_{3}
\end{array}\right\}
$$

where $N_{A}$ is the Avogadro's number. For the surface dipolar coverage oriented towards (opposed) the metal, we have: 


$$
\begin{gathered}
\text { anode } \\
\stackrel{\leftarrow}{\theta}=\frac{a}{2} e^{\mp X} /\left(\frac{1}{\Theta_{\mathrm{H}_{2} \mathrm{O}}}+\frac{\theta_{\mathrm{Hs}}}{\theta_{s}} \frac{1}{\Theta_{\mathrm{H}_{2} \mathrm{O}}}+a \operatorname{Cosh}[X]\right), \stackrel{\vec{t}}{\theta}=\frac{a}{2} e^{\mp X} /\left(\frac{1}{\Theta_{\mathrm{H}_{2} \mathrm{O}}}+\frac{\theta_{\mathrm{O}_{2} \mathrm{Hs}}+\theta_{\mathrm{OHs}}}{\theta_{s}} \frac{1}{\Theta_{\mathrm{H}_{2} \mathrm{O}}}+a \operatorname{Cosh}[X]\right)
\end{gathered}
$$

and using (13) with $\theta_{s}+\theta_{H s}+\vec{\theta}+\overleftarrow{\theta}=1$ (respectively $\theta_{s}+\theta_{O_{2} H s}+\theta_{O H s}+\vec{\theta}+\overleftarrow{\theta}=1$ ), we compute $\theta_{s}$ and $n_{s}$. The charge surface density $\sigma$ is calculated through the charge conservation law in the metal/electrolyte interface [6-8] [15] (Fig.2a) which is,

$$
\begin{aligned}
& J(r, t)-J_{F a r}(r, t)=J(r, t)-F\left(v_{H E Y}+v_{V O L}\right)=-\partial \sigma / \partial t \quad \text { (anode) } \\
& J(r, t)-J_{F a r}(r, t)=J(r, t)-F\left(v_{1}+v_{3}\right)=\partial \sigma / \partial t \quad \text { (cathode) }
\end{aligned}
$$

where $J_{F a r}$ is the faradaic current density.

The boundary condition for equation (5) at $x=L$, is obtained using the Gauss' theorem through the interface [15] $\partial \phi / \partial x(r, L, t)=-\sigma / \varepsilon_{C C}: \phi(r, L, t)$ is then calculated. Finally we calculate $\psi(r, t)$ using (6) and (10). $C_{H^{+}}(r, x=0, t), C_{H_{2}}(r, x=0, t)$ and $\phi(r, x=0, t)$ are calculated by the microscopic model.

The boundary conditions for (1) at $x=L$ (or $z=e_{N I}$ ) are $J_{H_{2}}(r, x=L, t)=-v_{T A F}-v_{H E Y}$ and $J_{O_{2}}(r, x=L, t)=-v_{1}$ (because the reactant concentration is computed constant through the nanometric diffuse layer thickness).

The multiscale model structure is based on a distributed-parameter Bond Graph description of the coupling between irreversible thermodynamics and electrodynamics, as we discuss elsewhere [8] [11-14]. In Fig. 3, we show one piece of the whole Bond Graph of our model (the complete Bond Graph description can be found in [8] [11]). The Bond Graph in Fig.3, represents, in the anode (the discussion for the cathode is analogous), the coupling between the diffuse layer, compact layer and electrochemical reactions models, where we show the causality chosen for our simulations. This Bond Graph is constituted by the diffuse layer sub-Bond Graphs, an element MC (representing the compact layer [8] [11]), two elements 0, one element 1 and one sub-Bond Graph (denoted Reaction) associated to the electrochemical reactions (11).

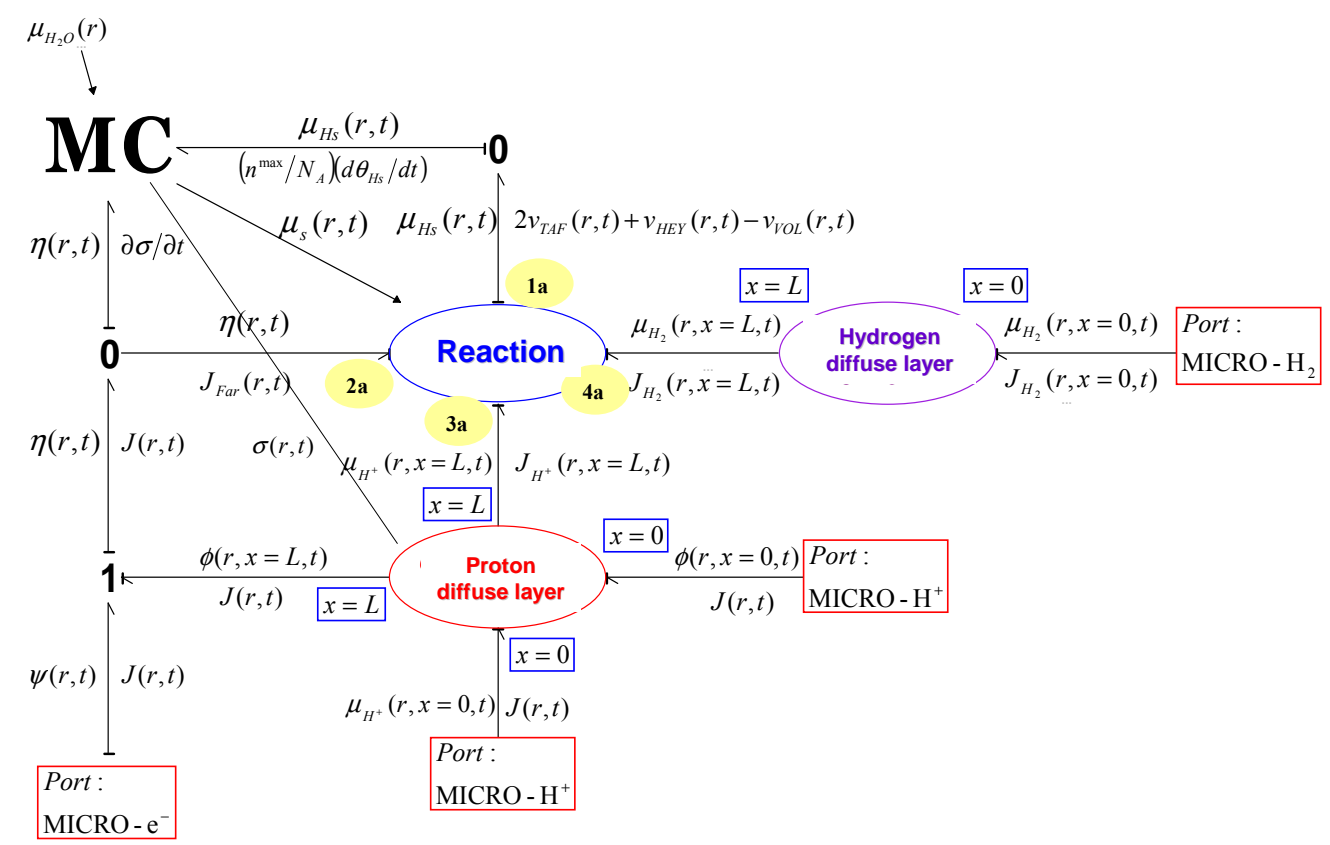

Fig. 3: The distributed-parameter Bond Graph representation of the anodic nanoscopic model where we show the causality chosen for our simulations.

For example, the element proton diffuse layer in Fig.3, has the associated distributed-parameter Bond Graph shown in Fig.4. This Bond Graph is composed by the connection of two sub-parts: a first one describing the protonic transport (I) and a second one describing the potential $\phi$ dynamics and its coupling 
with the proton accumulation (III). These sub-parts are linked through a power-conserving element TF (II) associated to a energy transfer coupling the efforts $\left(E(r, x, t)=-\nabla_{x} \phi\right.$ and $\left.F E(r, x, t)\right)$ between them.

The sub-part (I) is constituted by the interconnection of four multi-port elements: $\mathbf{0}, \mathbf{R}, \mathbf{1}, \mathbf{0}$ et $\mathbf{D T F}$. The element $\mathbf{0}$ represents the balance equation in (4) and the assumption of local equilibrium of the irreversible thermodynamics. The accumulation of protons is included in the element $\mathbf{C l}$. The element $\mathbf{R}$ represents the protonic transport in the diffuse layer, defined by the energy dissipative relation in (4) relating the two conjugated variables $\left(J_{H^{+}}, \nabla_{x} \widetilde{\mu}_{H^{+}}\right)$(the module associated to this element is here $\left.R T / D C_{H^{+}}\right)$. The junction $\mathbf{1}$, ensures the respect of the sign convention (flux opposed to the thermodynamic effort) and the effort balance (from the expression of the electrochemical potential): $-\nabla_{x} \widetilde{\mu}_{H^{+}}=-\nabla_{x} \mu_{H^{+}}-F \nabla_{x} \phi=-\nabla_{x} \mu_{H^{+}}+F E$. Always in the sub-part (I), the element DTF represents the interconnection between the energy accumulative (element $\mathbf{C l}$ in the sub-part (III)) and the dissipative (element R) parts. It combines the two adjoint differential relations: the thermodynamic force expressed as the gradient of the protonic electrochemical potential $\nabla_{x} \widetilde{\mu}_{H^{+}}$and the conservation law through the divergence of the flux $\nabla_{x} . J_{H^{+}}$. At the boundaries of this DTF we have: at $x=L$ (diffuse layer/compact layer boundary), the protonic molar flux $J_{H^{+}}(r, x=L, t)$ is imposed by the reaction model at the level of the compact layer $\left(J_{H^{+}}(r, x=L, t)=\left(v_{H E Y}+v_{V O L}\right)\right.$ in the anode, $J_{H^{+}}(r, x=L, t)=\left(v_{1}+v_{3}\right)$ in the cathode); at $x=0$ (port noted :1), the coupling between the nano and microscales takes place through two relations: firstly, according to the electroneutrality assumption at the microscale we have $\mu_{H^{+}}(r, x=0, t)^{N A N O}=\mu_{H^{+}}(r, t)^{M I C R O}=\mu_{0}+R T \ln C_{F I X^{-}}$which represents the equality of the chemical potentials at the boundary between the two scales. Secondly, the continuity of the molar fluxes at the boundary between the two scales $S_{H^{+}}(r, t)=J(r, t) \gamma \quad\left(J_{H^{+}}(r, x=0, t)=J(r, t)\right.$, as a consequence of the global current conservation). These two relations define a power-conserving connection element between the scales, noted by coupling in Fig.4b. Sub-part (III) is discussed elsewhere [8] [11].

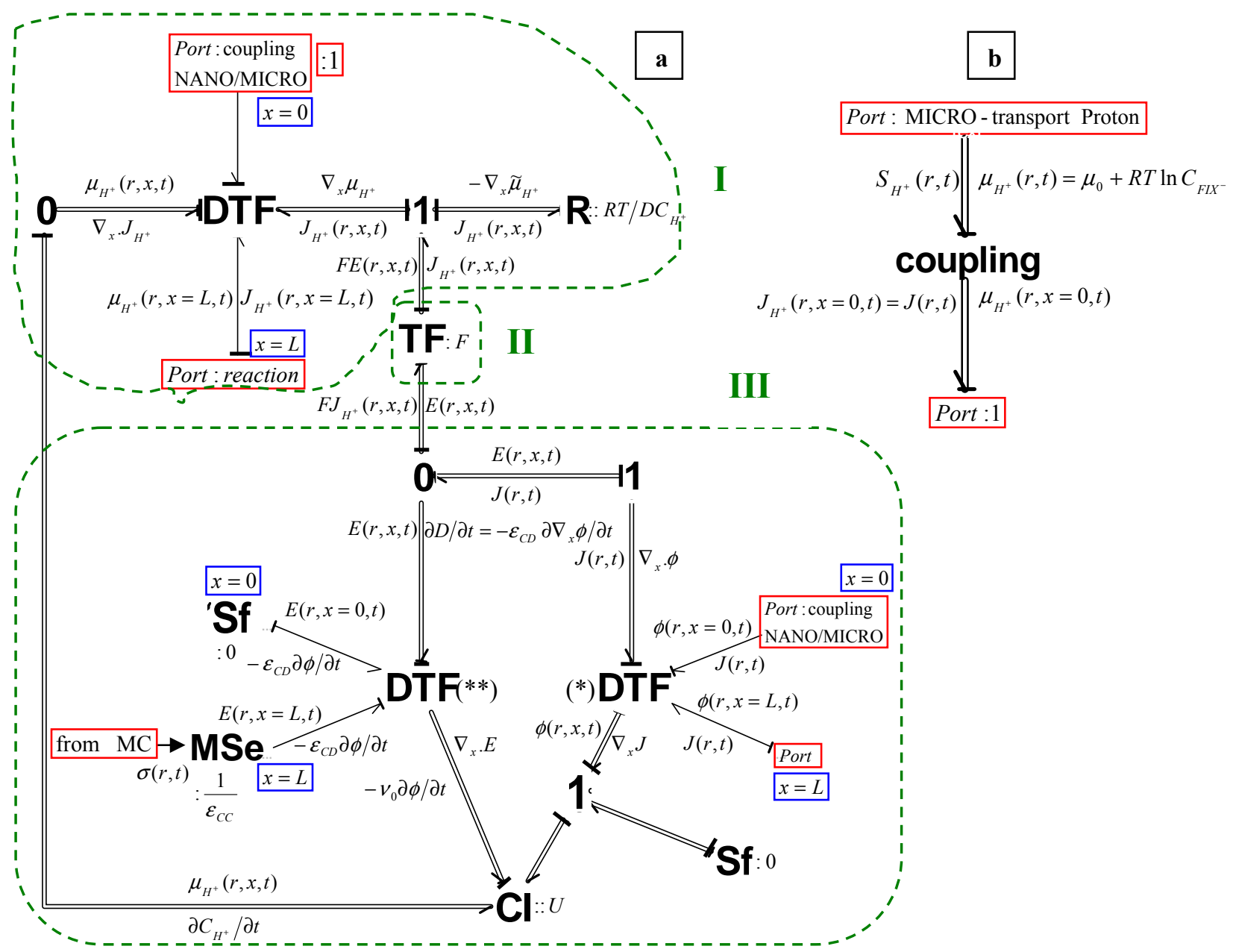

Fig. 4: a) The distributed parameter Bond Graph representation of the proton transport dynamic model in the diffuse layer (nanoscale). b) Element coupling between the protonic micro and nanoscales sub-models. 
The use of the Bond Graph language has allowed to hierarchize this multiscale model and to formulate it in modular way: a reticulated combination of conservation and constitutive equations. That means that it can be easily adapted to other electrochemical systems (solid oxide fuel cells, Lithium-ion batteries...) or coupled to another physico-chemical phenomena (ageing mechanisms, water transport...[8] [11-15]).

\section{Some simulations results}

The model was implemented under Femlab ${ }^{\circledR} /$ Simulink $^{\circledR} /$ Matlab $^{\circledR}$ using the finite element method, and the simulations have been performed on an Intel ${ }^{\circledR}$ Pentium ${ }^{\circledR} 4$ processor $2 \mathrm{GHZ}, 1$ Go of RAM. The used physicochemical parameters for the simulations shown here are [8]: $\gamma=5.59 \times 10^{7}\left(\mathrm{~m}^{2} / \mathrm{m}^{3}\right) ; e_{M}=50 \times 10^{-6}$, $e_{N I}=10^{-7}, e_{C A}=15 \times 10^{-6}(\mathrm{~m}) ; S_{M E A}=2.1 \times 10^{-4}\left(\mathrm{~m}^{2}\right) ; L=10^{-9}(\mathrm{~m}) ; C_{F I X}=1200\left(\mathrm{~mol} / \mathrm{m}^{3}\right) ; \alpha_{H E Y}=\alpha_{V O L}=0.5 . \alpha_{1}=0.8$, $\alpha_{3}=0.99, \quad \Delta G_{C}^{0}=1 \quad(\mathrm{~kJ} / \mathrm{mol}) ; \quad k_{T A F}=10^{-1} \quad k_{H E Y}=10^{-8} \quad k_{-H E Y}=10^{-6} k_{-V O L}=10^{-5} \quad k_{3}=10^{8} \quad(\mathrm{~m} / \mathrm{sec}) ; \quad k_{-T A F}=10^{-1}$ $k_{V O L}=10^{-2} \quad k_{-1}=10^{-5} \quad k_{2}=10^{8} \quad k_{-2}=10^{-2} \quad k_{-3}=1 \quad\left(\mathrm{~mol} /\left(\mathrm{m}^{2} . \mathrm{sec}\right)\right) ; \quad k_{1}=10^{4} \quad\left(\mathrm{~m}^{4} /(\mathrm{mol} . \mathrm{sec})\right) ; \quad \varepsilon_{C C A}=6 \times \varepsilon_{0}$, $\varepsilon_{C C C}=4 \times \varepsilon_{0}, \varepsilon_{C D A}=\varepsilon_{C D C}=20 \times \varepsilon_{0}(\mathrm{C} /($ Volt.m) $) ; \lambda=14$. The empirical relations used here [7-8] [15]:

- $C_{H_{2}}(z=0, t)=\frac{P_{A}-p_{\text {sat }}}{1.09 \times 10^{5} \times \exp (77 / T)}, C_{O_{2}}(z=0, t)=\frac{P_{C}-p_{\text {sat }}}{5.08 \times 10^{5} \times \exp (-498 / T)}$ (Henry's laws) with

$p_{\text {sat }}=10^{-5} \times \exp \left(23.1961-\frac{3816.44}{T-46.13}\right)$

(bars)

- $\Theta_{H_{2} O}=1.435+0.0022 \lambda-\frac{2.75}{\lambda}-0.13 \ln \lambda$;

- $D_{H+}=2.97 \times 10^{-14} \times T \times 10^{\left[\frac{1.33 \times(293-T)-0.001 \times(293-T)^{2}}{T-168}\right]+3}=g_{H^{+}} / f C_{F I X}\left(\mathrm{~m}^{2} / \mathrm{sec}\right)$

- $D_{H_{2}}=3.92 \times 10^{-14} \times \exp (0.025 \times T)\left(\mathrm{m}^{2} / \mathrm{sec}\right)$

- $\mu_{i}(p, T)=\mu_{i 0}(T)+R T \ln a_{i}$ (thermodynamic property) where $a_{i}$ is the activity (for ideal solutions -our assumption-: $\left.a_{i} \cong C_{i}\right)$.

The dynamic PEFC potential $U_{\text {cell }}(t)$, as response to a current perturbation $I(t)$, is calculated through:

$$
U_{\text {cell }}(t)=\psi_{C}(r=0, t)-\psi_{A}(r=0, t)
$$

In Fig. 5 we show the sensitivity of the simulated MEA polarisation curve a to the Nafion ${ }^{\circledR}$ and platinum loadings: the increase of the Nafion ${ }^{\circledR}$ content leads to the diminution of the limit current (because of the increase of the transport limitations). Our model allows to compute optimal loadings values in good agreement with experimental results as we show elsewhere [8].

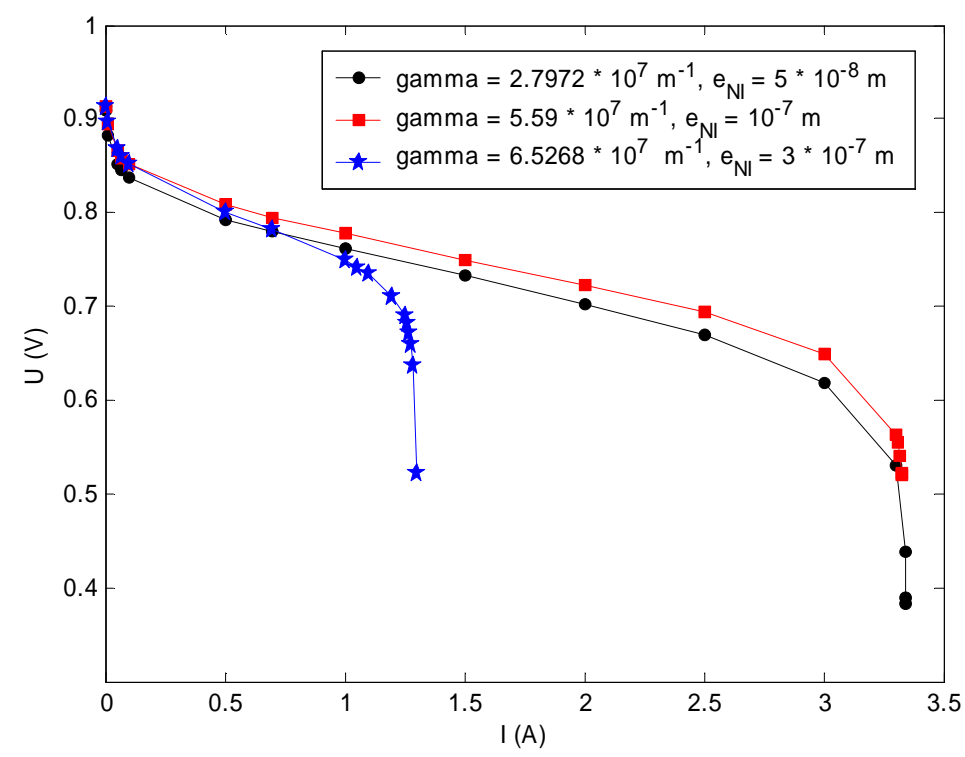

Fig. 5: Sensitivity of the simulated polarisation curve to the electrodes Nafion ${ }^{\circledR}$ and platinum loading ( $T=353$ $\mathrm{K}, P_{A}=P_{C}=1.5$ bar). 
Based on time simulations, the frequency response and the impedance spectrum of the system can be derived (Fast Fourier Transform on the input and output signals). A small sinusoidal signal (e.g. $15 \mathrm{~mA}$ ) has been superimposed on the fixed (DC) current level $\bar{I}$ for frequencies ranging from $0.01 \mathrm{~Hz}$ to $10^{6} \mathrm{~Hz}$.

In Fig. 6 we show a comparison between the sensitivity of experimental and simulated MEA impedance spectroscopy to the Nafion ${ }^{\circledR}$ and platinum loadings. Our model is in good qualitative agreement with experimental results, as discussed by Franco in [8]. For each nominal current value, the amplitude of the arc strong depends on the thickness of the impregnated Nafion ${ }^{\circledR}$ layer $e_{N I}$ and on the specific catalyst area $\gamma$. For the quantitative agreement we have now started a parametric identification study of our model, focused on the Damjanovic mechanism [8].
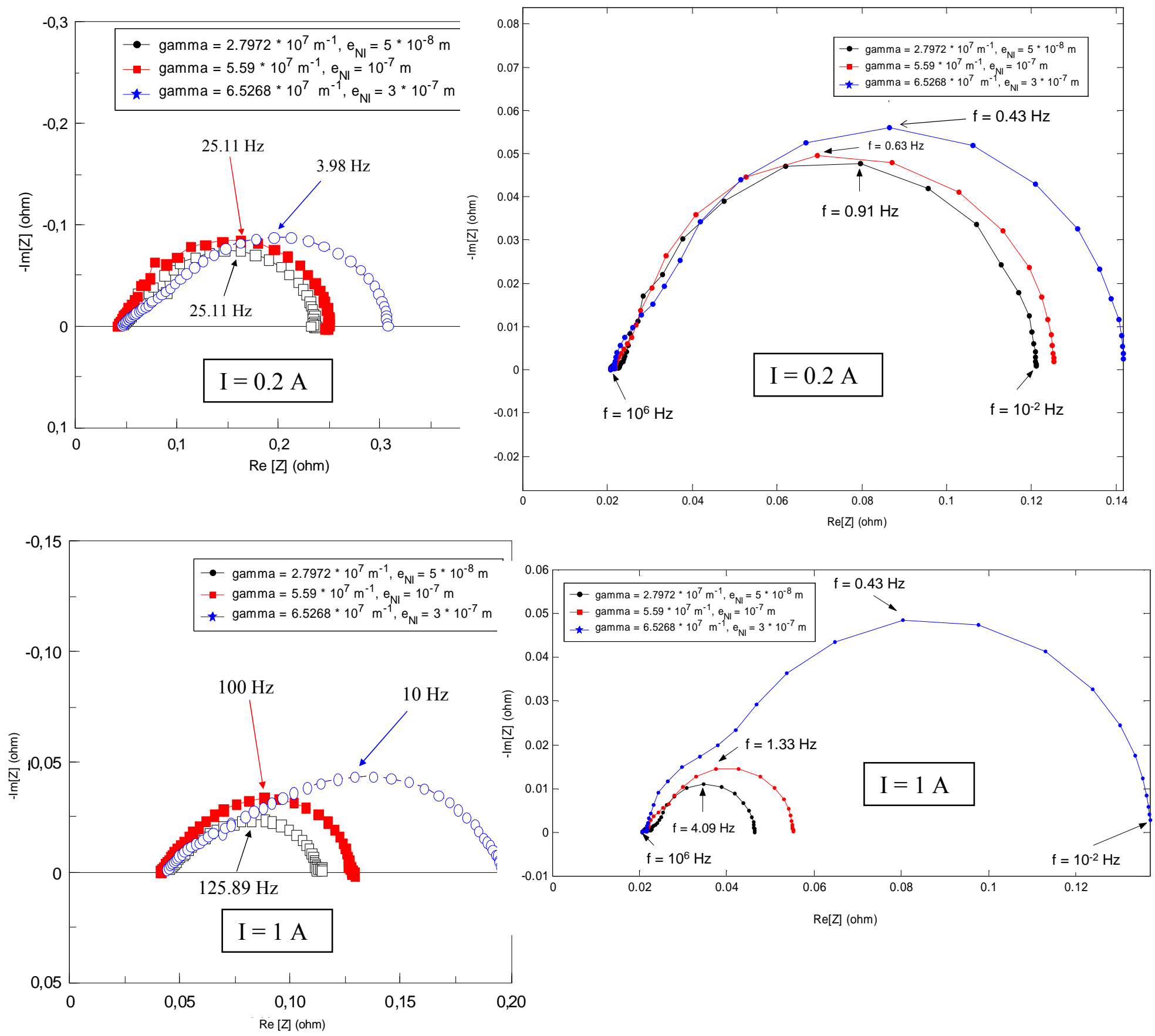

Figure 6: Comparison between simulated EIS spectra sensitivity to the electrodes $\mathrm{Nafion}^{\circledR}$ and platinum loadings $\left(T=353 \mathrm{~K}, P_{A}=P_{C}=1.5\right.$ bar, $\bar{I}=0.2$ and $\left.\bar{I}=1 \mathrm{~A}\right)$ and experiments (performed in a PEFC with $S_{M E A}=2.1 \times 10^{-4} \mathrm{~m}^{2}$, as discussed by Franco in [8]). 
The Fig.7 shows the influence of the nominal current $\bar{I}$ on the EIS. The increase of the demanded current leads to the diminution of the amplitude of the arcs, because of the orientation of water dipoles on the catalyst, improving electron transfer, as discussed in [8]: the capacitive effect diminishes, in accordance with well know experimental results [3-5] [8] (Fig. 7a and 7b). For larger nominal current values, the amplitude increases (because of the transport limitations) (Fig. 7c). Similar studies can be performed for temperature and pressures sensitivity, and other dynamical PEFC operations (current steps, cyclic voltammetry...) can be simulated [6] [8].

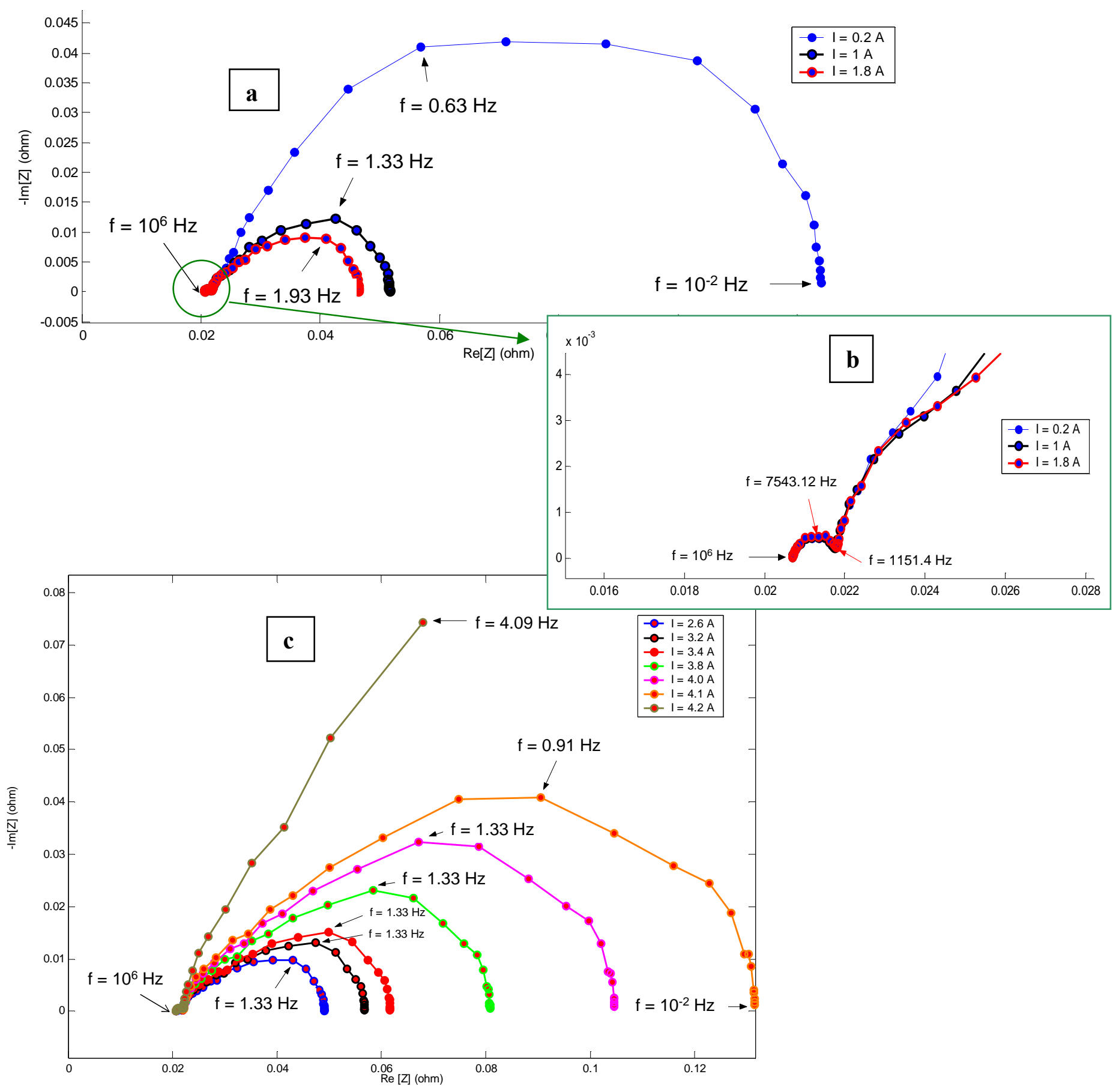

Figure 7: Simulated EIS spectra sensitivity to the nominal current $\bar{I}\left(T=353 \mathrm{~K}, P_{A}=P_{C}=1.5\right.$ bar).

\section{Concluding Remarks}

In this paper we propose a new mechanistic model for the dynamic simulation of a polymer electrolyte fuel cell Membrane-Electrodes Assembly. Our model results of the coupling of a microscale transport phenomena description through the electrodes and the electrolyte thickness, a spatially distributed microscale model of the reactant diffusion through the Nafion ${ }^{\circledR}$ layer covering the $\mathrm{Pt} / \mathrm{C}$ particles, and a 
spatially distributed nanoscopic description of the Nafion ${ }^{\Theta}-\mathrm{Pt} / \mathrm{C}$ interface. This interfacial model is based on an internal description of the double layer dynamics, coupling the transport phenomena in the diffuse layer and the electrochemical reactions in the compact layer.

The model is based on a distributed-parameter Bond Graph description of the coupling between irreversible thermodynamics and electrodynamics. The use of the Bond Graph language has allowed to hierarchize this multiscale model and to formulate it in modular way, so that it is reusable and could be adapted to other electrochemical systems (solid oxide fuel cells, Lithium-ion batteries...) or coupled to another physicochemical phenomena (ageing mechanisms, water transport...[6] [8] [11-15]).

Our model is mainly dedicated to the diagnostics of the dynamic fuel cell operation, but it can help also to improve electrodes performance and durability (through the optimisation of platinum/Nafion ${ }^{\circledR}$ loadings, and their volumetric distribution) [8]. Furthermore, the model can evaluate the contribution of different physicochemical phenomena into the PEFC performance. Unlike classical analogical models based on analogy electric circuits, our approach allows to predict the influence of working conditions on the impedance spectra characteristics. The sensitivity of the simulations results to inputs like nominal current, temperature, reactant pressure and electrodes $\mathrm{Nafion}^{\circledR}$ and platinum loadings are in good qualitative agreement with experimental results. We have now started a parametric identification study of our model [8].

\section{References}

[1] Eikerling M., Kornyshev A.A. and Kulikovsky A., Can theory help to improve fuel cells?; The Fuel Cell Review (December 2004-January 2005).

[2] Berthier F., Diard J.-P. and Michel R., Distinguishability of equivalent circuits containing CPEs. Part I. Theoretical part; Journal of Electroanalytical Chemistry 510 (2001) 1-11.

[3] Walkiewicz S., Bautista M., Bultel Y., Diard J.-P. Electrochemical impedance spectroscopy study of the $P E M F C$ behaviour (original title: Etude par spectroscopie d'impédance électrochimique du comportement en fonctionnement d'une PEMFC); 14éme Forum sur les Impédances Electrochimiques (Paris, January 14, 2002).

[4] Kuhn H., Andreaus B., Wokaun A. and Scherer G.G.; Electrochimica Acta, 51 Issues 8-9 (2006) 16221628.

[5] Kuhn H., Andreaus B., Wokaun A. and Scherer G.G.; in Proceedings of $3^{\text {rd }}$ European PEFC Forum, Luzern, Switzerland, (2005) paper no. \#B076 (in conference CD).

[6] Franco A.A., Schott P., Jallut C. and Maschke B.M., A Multiscale Dynamic Mechanistic Model for Transient Analysis of Electrochemical Cells. Orally presented in Symposium "T1: Modeling of Electrochemical Systems" $207^{\text {th }}$ Meeting of the Electrochemical Society (Québec, May 15-20), abstract \#1296 (2005).

[7] Franco A.A., Schott P., Jallut C. and Maschke B.M.; A Multiscale Dynamic Mechanistic Model for Transient Analysis of PEFCs. In: Proc. $3^{\text {rd }}$ European PEFC Forum, Luzern, 2005, paper \#B063 (in conference CD) (2005).

[8] Franco A.A., A Physical Multiscale Model of the Electrochemical Dynamics in a Polymer Electrolyte Fuel Cell (Original title: Un modèle physique multiéchelle de la dynamique électrochimique dans une pile à combustible à électrolyte polymère), PhD Thesis Université Claude Bernard Lyon-1 (France) no. 2005LYO10239 (2005).

[9] Van der Schaft A.J. and Maschke B.M., Compositional modelling of distributed-parameter systems; In: Advanced topics in control systems theory, Chapter 4, pp.115-154, Springer (2004).

[10] Eberard D., Lefèvre L. and Maschke B.M., Multiscale coupling in heterogeneous diffusion processes: a port-based approach, Proc. International Conference PhysCon 2005, August 24-26, 2005, Saint Petersburg, Russia.

[11] Franco A.A., Schott P., Jallut C., Maschke B., Multi-scale Bond Graph model of the Electrochemical Dynamics in a PEFC; orally presented and in Proceedings of the $5^{\text {th }}$ MATHMOD Conference, Vienna, February 8-10, 2006 (ISBN 3-901608-25-7) (page \# 93 and in conference CD) (2006).

[12] Franco A. A., Schott P., Jallut C., Maschke B.; A Multiscale Dynamic Model of a PEFC Electrode (Original title: Un Modèle Multiéchelle Dynamique d'une Electrode de PEFC); e-STA (e-revue, http://www.see.asso.fr/) 2 (2005) no.3.

[13] Franco A.A.; A Physical multiscale model for transient analysis of electrochemical cells: an infinitedimensional Bond Graph approach. Finalist for the award "Young Scientist Award 2005" of the Solid State Ionics Conference (SSI-15, Baden-Baden, 17-22 July 2005), and orally presented, abstract \#9 (2005).

[14] Franco A.A., P. Schott, C. Jallut and B. Maschke; A Multiscale Dynamic Model of a PEFC electrode (Original title: Un Modèle Multiéchelle Dynamique d'une Electrode de PEFC). Orally presented and in Proceedings of the JDMACS 2005 (Lyon, 5-9 September 2005) (in conference CD) (2005).

[15] Franco A.A., Schott P., Jallut C., Maschke B., A Dynamic Mechanistic Model of an Electrochemical Interface; Journal of the Electrochemical Society, 153 (6) A1053-A1061 (2006). 